\title{
The "bespoke" recovery: Available when the tailoring is guided by experience and high-quality data
}

\author{
W. Paul Murphy, MD, and John F. Butterworth IV, MD
}

\footnotetext{
From the Department of Anesthesiology, Virginia Commonwealth University School of Medicine, VCU Health System, Richmond, Va.

Disclosures: Authors have nothing to disclose with regard to commercial support.

Received for publication June 27, 2017; accepted for publication July 25, 2017; available ahead of print September 6, 2017.

Address for reprints: John F. Butterworth IV, MD, Department of Anesthesiology, Virginia Commonwealth University School of Medicine, VCU Health System, 1200 E Broad St, Richmond, VA 23298-0695 (E-mail: jfbivjbutter@yahoo.com).

J Thorac Cardiovasc Surg 2017;154:1666-7

$0022-5223 / \$ 36.00$

Copyright (c) 2017 by The American Association for Thoracic Surgery

http://dx.doi.org/10.1016/j.jtcvs.2017.07.040
}

As we are guided by bigger data sets and smaller budgets, we still must consider the entire patient in context when we tailor our clinical decisions toward higher quality, lower cost, and more personalized care. Two new models proposed by Subramaniam and colleagues ${ }^{1}$ in their article in this issue of the Journal explore variables that are associated with successful immediate extubation in the operating room after a heterogenous group of cardiac procedures. The first model uses preoperative and surgical variables, to which the second adds anesthetic choices. The models support the routine pursuit of immediate extubation in most cardiac surgical patients while identifying those patients with comorbidities and other predictors of unsuccessful extubation.

We, along with Subramaniam and colleagues, ${ }^{1}$ acknowledge that some clinical choices associated with success may have been made by clinicians because of a suspicion that a given patient was a strong candidate for immediate extubation. Some of the criteria seem obvious (eg, albumin concentration, age, need for home oxygen), others less so. Individual numbers and histories, however, do not tell the entire story. Those successfully extubated included more smokers, more committed drinkers, the most anemic patient (hematocrit of $13.5 \%$ ), and the patient with the lowest preoperative left ventricular ejection fraction $(5 \%$ - and that patient didn't get a transplant!). Although an anemic, harddrinking patient with a low ejection fraction seems unlikely to do well with immediate extubation after heart surgery, Subramaniam and colleagues ${ }^{1}$ remind us to consider the whole patient and that higher quality, lower cost, individualized care is appropriate for all patients. These patients benefited from higher cost but data-supported techniques, such as intrathecal morphine (for thoracotomy approaches), intravenous acetaminophen, and remifentanil, as well as a standardized anesthetic with vigilant monitoring of neuromuscular blockade.

Years ago, one of us was taken by his father to purchase his first blazer. The tailor, hair white from chalk and experience, took one look and said, "Good afternoon, 42 regular, come

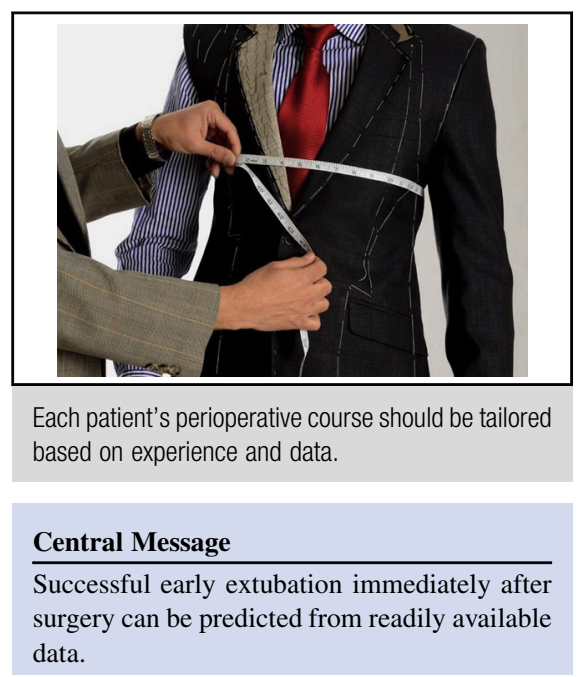

See Article page 1656. with me." The tailor's experience provided a start, then he adjusted one of his available jackets to a perfect fit. We need much the same approach in medicine, where the stakes are much higher than one's appearance at a high school dance.

More than 50 years ago, Lefemine and Harken ${ }^{2}$ showed that routine postoperative ventilation improved outcomes after cardiac surgery. It is important to remember, however, that Lefemine and Harken ${ }^{2}$ were comparing routine extubation with routine controlled ventilation, rather than comparing selective extubation with routine ventilation. In other words, Subramanian and colleagues ${ }^{1}$ do not disagree with Lefemine and Harken ${ }^{2}$ ! By proposing objective predictors of successful immediate extubation, Subramaniam and colleagues ${ }^{1}$ help clinicians identify what is best for their individual patients. These results, consistent with the conclusions of others regarding successful fast-track cardiac protocols and with the burgeoning "Enhanced Recovery After Surgery" literature, offer strong support for data-driven care.

Transitions of care represent major opportunities for improvement. When should one move a patient from home to a skilled nursing facility, an acute care hospital, or an intensive care unit bed? When may that patient's care be de-escalated safely? Choosing when to invest (or forego) additional resources remains one of the most difficult aspects of modern medical care. Subramanian 
and colleagues ${ }^{1}$ propose a means by which to minimize the scale and duration of a costly and invasive treatment: intubation with mechanical ventilation. They remind us that our choices in the operating room may have far-reaching effects as we pursue higher quality, lower costs and individualized care for our patients.

\section{References}

1. Subramaniam K, DeAndrade D, Mandell D, Althouse AD, Manmohan R, Esper SA, et al. Predictors of operating room extubation in adult cardiac surgery. J Thorac Cardiovasc Surg. 2017;154:1656-65.

2. Lefemine AA, Harken DE. Postoperative care following open-heart operations: routine use of controlled ventilation. J Thorac Cardiovasc Surg. 1966;52:207-16.
3. Myles PS, Daly DJ, Djaiani G, Lee A, Cheng DC. A systematic review of the safety and effectiveness of fast-track cardiac anesthesia. Anesthesiology. 2003; 99:982-7.

4. Wong DT, Cheng DC, Kustra R, Tibshirani R, Karski J, Carroll-Munro J, et al Risk factors of delayed extubation, prolonged length of stay in the intensive care unit, and mortality in patients undergoing coronary artery bypass graft with fast-track cardiac anesthesia: a new cardiac risk score. Anesthesiology. 1999;91: 936-44.

5. Ljungqvist O, Scott M, Fearon KC. Enhanced recovery after surgery: a review. JAMA Surg. 2017;152:292-8.

6. Butterworth J, James R, Prielipp RC, Cerese J, Livingston J, Burnett DA. Do shorter-acting neuromuscular blocking drugs or opioids associate with reduced intensive care unit or hospital lengths of stay after coronary artery bypass surgery? CABG Clinical Benchmarking Data Base Participants. Anesthesiology. 1998;88: 1437-46. 